\title{
Field Theory with an External Potential
}

\author{
W. J. M. A. Hochstenbach \\ Institut für Theoretische Physik, Universität Göttingen, D-3400 Göttingen, \\ Federal Republic of Germany
}

\begin{abstract}
A quantum theory for charged spin zero particles interacting with an external potential is constructed for a certain class of time-independent potentials.

For potentials of a different class, a group of Bogoliubov transformations generated by the solutions of a classical differential equation with an external potential is defined in the free one-particle space. We give necessary and sufficient conditions on the potential for this group to be unitarily implementable in the Fock space of the free field.
\end{abstract}

\section{Introduction}

Although perturbation theory constitutes the basis for the practical calculations in quantum electrodynamics, a direct justification for applying perturbation theory is prevented by the existence of a Euclidean symmetry group. Generally one tries to circumvent this implication of Haag's theorem by breaking the symmetry.

For charged spin zero particles in an external time-independent potential we prove in Section 3 a conjecture of Schroer, Seiler and Swieca [1]: the interaction Hamiltonian does not exist in the Fock space of the free field if the external potential contains a three-vector part. Thus, in a field theory with an external, time-independent vector potential $(0, \boldsymbol{A})$ the assertion of Haag's theorem is valid, although its assumptions are not fulfilled.

To be more precise, let $\left(A^{\mu}\right)(\mu=0,1,2,3)$ be an element of the function class 2 specified in Section 3; the time evolution of the interacting field in the Fock space of the free particles is determined by a one-parameter group of Bogoliubov transformations in the free one-particle space; at time $t=0$ the interacting field coincides with the free field. Then the time evolution is described by a strongly continuous, one-parameter group of unitary operators in the Fock space of the free field, if and only if $A=0$ on $\mathbb{R}^{3}$ and

$$
Q_{0}(t)=\int \frac{d \boldsymbol{p} d \boldsymbol{p}^{\prime}}{\omega(\boldsymbol{p}) \omega\left(\boldsymbol{p}^{\prime}\right)}\left(\frac{\omega(\boldsymbol{p})-\omega\left(\boldsymbol{p}^{\prime}\right)}{\omega(\boldsymbol{p})+\omega\left(\boldsymbol{p}^{\prime}\right)}\right)^{2}\left|\tilde{A}_{0}\left(\boldsymbol{p}-\boldsymbol{p}^{\prime}\right)\right|^{2} \sin ^{2} \frac{\left(\omega(\boldsymbol{p})+\omega\left(\boldsymbol{p}^{\prime}\right)\right) t}{2}
$$

is finite for all times $t$ and continuous in $t$ at 0 . 
In the spin zero case, the vanishing of $\boldsymbol{A}$ stems from the presence of derivatives in the interaction part of the Hamiltonian in the free one-particle space.

In Section 2 we show the existence of a non-trivial class of potentials, for which one can construct a Fock quantization with a unique vacuum for charged spin zero particles in an external potential. Furthermore, it is shown that every quantization with a unique vacuum, whose generating functional is in the Fock sector, is unitarily equivalent to the Fock quantization.

\section{Quantization of the Klein-Gordon Equation with an External Potential}

Our main concern will be proving the existence of a non-trivial class $\mathscr{P}$ of potentials, for which one can define a one-particle Hilbert space $\mathscr{H}_{P}$ and a one-parameter strongly continuous group with a strictly positive generator $\overline{|B|}$.

Let $\mathscr{H}_{E_{0}}$ be the direct sum of the Sobolev space $W^{1}\left(\mathbb{R}^{3}\right)$ and the Hilbert space $L^{2}\left(\mathbb{R}^{3}\right)$. The operator $B_{0}$ in $\mathscr{H}_{E_{0}}$, with domain $D\left(B_{0}\right)=C_{0}^{\infty}\left(\mathbb{R}^{3}\right) \oplus C_{0}^{\infty}\left(\mathbb{R}^{3}\right)$ and defined by $B_{0}=\left(\begin{array}{cc}0 & 1 \\ -\Delta+m^{2} & 0\end{array}\right)$ on $D\left(B_{0}\right)(m \neq 0)$, is essentially self-adjoint on $D\left(B_{0}\right)$.

Its closure $\bar{B}_{0}$ has domain $D\left(\bar{B}_{0}\right)=W^{2}\left(\mathbb{R}^{3}\right) \oplus W^{1}\left(\mathbb{R}^{3}\right)$, and is given by $\bar{B}_{0}=\left(\begin{array}{cc}0 & 1 \\ \omega^{2} & 0\end{array}\right)$, where $\omega^{2}$ is the closure of the operator $-\Delta+m^{2}$ on $C_{0}^{\infty}\left(\mathbb{R}^{3}\right)$ as a mapping from $W^{1}\left(\mathbb{R}^{3}\right)$ to $L^{2}\left(\mathbb{R}^{3}\right)$. We close the operator $B_{0}$ and denote this closure again by $B_{0}$. The self-adjoint operator $B_{0}$ has the property that $B_{0}^{-1}$ is bounded with $\left\|B_{0}^{-1}\right\|=m^{-1}$. In the space $\mathscr{H}_{E_{0}}$ we introduce the perturbation $B_{\text {int }}:=\left(\begin{array}{cc}e A_{0} & 0 \\ e^{2} A^{2}+i e \nabla \cdot A+2 i e A \cdot \nabla & e A_{0}\end{array}\right)$ as a bounded operator for potentials $\left(A^{\mu}\right)$ $(\mu=0,1,2,3)$ in $C_{B}^{1}\left(\mathbb{R}^{3}\right)$, the class of real bounded continuous functions with bounded and continuous first partial derivatives.

For potentials in $C_{B}^{1}\left(\mathbb{R}^{3}\right)$ with the additional property $\left|e A_{0}\right| \leqq m$ an inner product $(\cdot, \cdot)_{E}$ is defined on $C_{0}^{\infty}\left(\mathbb{R}^{3}\right) \oplus C_{0}^{\infty}\left(\mathbb{R}^{3}\right)$ by

$$
\begin{aligned}
(f, g)_{E}= & \int\left\{\left((-i \nabla-e A) f_{1}\right) *(-i \nabla-e A) g_{1}+\left(m^{2}-e^{2} A_{0}^{2}\right) f_{1}^{*} g_{1}\right. \\
& \left.+\left(e A_{0} f_{1}+f_{2}\right)^{*}\left(e A_{0} g_{1}+g_{2}\right)\right\} d \boldsymbol{x}
\end{aligned}
$$

for all $f, g \in C_{0}^{\infty}\left(\mathbb{R}^{3}\right) \oplus C_{0}^{\infty}\left(\mathbb{R}^{3}\right)$.

The completion of $C_{0}^{\infty}\left(\mathbb{R}^{3}\right) \oplus C_{0}^{\infty}\left(\mathbb{R}^{3}\right)$ in the energy norm is denoted by $\mathscr{H}_{E}$; the Hilbert spaces $\mathscr{H}_{E_{0}}$ and $\mathscr{H}_{E}$ are related as sets by $\mathscr{H}_{E_{0}} \subset \mathscr{H}_{E}$. In the same way as one proves the Kato-Rellich theorem, one obtains the following result.

Lemma 2.1. If $\left(A^{\mu}\right) \in C_{B}^{1}\left(\mathbb{R}^{3}\right)$ and $\left|e A_{0}\right| \leqq m$, then the operator $B:=B_{0}+B_{\text {int }}$ is essentially self-adjoint on $D\left(B_{0}\right)$ in the Hilbert space $\mathscr{H}_{E}$.

If one assumes that the norms $\|\cdot\|_{E_{0}}$ and $\|\cdot\|_{E}$ on $C_{0}^{\infty}\left(\mathbb{R}^{3}\right) \oplus C_{0}^{\infty}\left(\mathbb{R}^{3}\right)$ are equivalent, one gets a stronger result: the operator $B$ is self-adjoint on $D\left(B_{0}\right)$ in $\mathscr{H}_{E} ; C_{0}^{\infty}\left(\mathbb{R}^{3}\right) \oplus C_{0}^{\infty}\left(\mathbb{R}^{3}\right)$ is a core for $B$.

Let $\mathscr{P}_{0}$ be the class of potentials $\left(A^{\mu}\right)$ with $A^{\mu} \in C_{B}^{1}\left(\mathbb{R}^{3}\right)$ and $\left|e A_{0}\right| \leqq m$ such that there exists $c>0$ with $\frac{1}{c}\|f\|_{E_{0}} \leqq\|f\|_{E} \leqq c\|f\|_{E_{0}}$ for all $f \in C_{0}^{\infty}\left(\mathbb{R}^{3}\right) \oplus C_{0}^{\infty}\left(\mathbb{R}^{3}\right)$. The class $\mathscr{P}_{0}$ is non-trivial; in fact one can show the following lemma. 
Lemma 2.2. If $A_{0}=0$ and $A^{i} \in C_{B}^{1}\left(\mathbb{R}^{3}\right)(i=1,2,3)$, then $\left(A^{\mu}\right) \in \mathscr{P}_{0}$. If $\left|e A_{0}\right| \leqq m$, $A_{0} \in C_{B}^{1}\left(\mathbb{R}^{3}\right)$ and $A_{0}(\boldsymbol{x})=O\left(|\boldsymbol{x}|^{-1-\varepsilon}\right)$ for $|\boldsymbol{x}| \rightarrow \infty(\varepsilon>0)$ and $A^{i}=0(i=1,2,3)$, then $\left(A^{\mu}\right) \in \mathscr{P}_{0}$.

To prove the second statement, one needs an estimate from [2]: if $A_{0}^{2} \in L^{2}\left(\mathbb{R}^{3}\right)$ and $A_{0}^{2}(\boldsymbol{x})=O\left(|\boldsymbol{x}|^{-2-\varepsilon}\right)$ for $|\boldsymbol{x}| \rightarrow \infty(\varepsilon>0)$, then there exists $c>0$, such that for all $f \in C_{0}^{\infty}\left(\mathbb{R}^{3}\right) \quad \int\left|e A_{0}(\boldsymbol{x}) f(\boldsymbol{x})\right|^{2} d \boldsymbol{x} \leqq c \int|\nabla f(\boldsymbol{x})|^{2} d \boldsymbol{x}$.

As a further restriction on the class of potentials, we only admit potentials in $\mathscr{P}$, the subset of $\mathscr{P}_{0}$ such that 0 is a point of the resolvent set $\varrho(B)$. The class $\mathscr{P}$ is non-trivial as the next lemma shows.

Lemma 2.3. For every $\left(A^{\mu}\right)$ in Lemma 2.2 there exists $r>0$, such that $\left(\alpha A^{\mu}\right) \in \mathscr{P}$ for all $\alpha \in \mathbb{R}$ with $|\alpha|<r$.

Proof. Range $(B)=\operatorname{Range}\left(B B_{0}^{-1}\right)=\operatorname{Range}\left(\mathbb{1}+B_{\text {int }} B_{0}^{-1}\right)=\mathscr{H}_{E}$ if $\left\|B_{\text {int }} B_{0}^{-1}\right\|<1$. There exists $c>0$ such that for all $\alpha$ in $\mathbb{R}$ with $|\alpha| \leqq 1,\left\|B_{\text {int }}\left(\left(\alpha A^{\mu}\right)\right) B_{0}^{-1}\right\| \leqq|\alpha| m c$. By choosing $\alpha$ sufficiently small Range $\left(B\left(\left(\alpha A^{\mu}\right)\right)\right)=\mathscr{H}_{E}$.

Let $V(\cdot)$ be the one-parameter strongly continuous unitary group, generated by the self-adjoint operator $B$ in $\mathscr{H}_{E} ; P_{+}$and $P_{-}$are the spectral projections of $B$, connected with the sets $(0, \infty)$ and $(-\infty, 0)$. Because of $\left(A^{\mu}\right) \in \mathscr{P}$ the bilinear functional $(\cdot, \cdot)_{Q}$ on $\mathscr{H}_{E}$, defined by $(f, g)_{Q}:=\left(f, B^{-1} g\right)_{E}$ for all $f, g \in \mathscr{H}_{E}$, is bounded;

$$
(f, g)_{Q}=\left(f_{1}, g_{2}\right)+\left(f_{2}, g_{1}\right) \text { and }(V(t) f, V(t) g)_{Q}=(f, g)_{Q}
$$

for all $f, g \in \mathscr{H}_{E}$ and for all $t \in \mathbb{R}$.

For potentials in $\mathscr{P}$ one can introduce a new inner product in $\mathscr{H}_{E}$; for all $f, g \in \mathscr{H}_{E},(f, g)_{I}:=\left(f,|B|^{-1} g\right)_{E}$. $\mathscr{H}_{I}$ denotes the completion of $\mathscr{H}_{E}$ with respect to the norm $\|\cdot\|_{I}$. The operators $V(t), P_{+}, P_{-}$and the bilinear functional $(\cdot, \cdot)_{Q}$ are $\mathscr{H}_{Y}$-bounded on $\mathscr{H}_{E}$; so one can extend them to bounded linear operators (resp. bilinear functional) on $\mathscr{H}_{I}$. We denote these extensions by the same symbols. The operator $B$ is essentially self-adjoint in $\mathscr{H}_{I}$; its closure $\bar{B}$ in $\mathscr{H}_{I}$ is the generator of the one-parameter strongly continuous unitary group $V(\cdot)$. The starting point for quantizing a classical equation $i \partial_{t} f(t)=\bar{B} f(t)$ in $\mathscr{H}_{I}$ is a triple $(L, \sigma, V(\cdot))$, where $(L, \sigma)$ is a symplectic space and $V(\cdot)$ a one-parameter group of symplectic transformations. We choose as the triple $L=\mathscr{H}_{I}^{r}$ (the underlying real linear space of the Hilbert space $\left.\mathscr{H}_{I}\right), \sigma(f, g)=\operatorname{Im}(f, g)_{Q}$ for all $f, g \in \mathscr{H}_{I}$ and $V(\cdot)$ the oneparameter strongly continuous group of unitary operators in $\mathscr{H}_{I}$.

From [3] we adopt the notion of quantization. A quantization over $(L, \sigma, V(\cdot))$ is a quadruple $(\mathscr{K}, W, \Omega, U(\cdot))$ such that 1$)(\mathscr{K}, W, \Omega)$ is a cyclic Weyl system over $(L, \sigma)$; 2) $t \mapsto U(t)$ is a one-parameter strongly continuous group of unitary operators on $\mathscr{K} ; U(t)=e^{-i H t}$ for all $t \in \mathbb{R}$ with $\left.H \geqq 0 ; 3\right)$ the symplectic transformations are unitarily implemented by the $U(t)$ 's and $U(t) \Omega=\Omega$ for all $t \in \mathbb{R} ; W(V(t) f)=$ $U(t) W(f) U(t)^{-1}$ for all $t \in \mathbb{R}$ and $f \in L$. Two quantizations $(\mathscr{K}, W, \Omega, U(\cdot))$ and $\left(\mathscr{K}_{0}, W_{0}, \Omega_{0}, U_{0}(\cdot)\right)$ over $(L, \sigma, V(\cdot))$ are unitarily equivalent, if there exists a unitary intertwiner $U$ for the cyclic Weyl systems $(\mathscr{K}, W, \Omega)$ and $\left(\mathscr{K}_{0}, W_{0}, \Omega_{0}\right)$.

Lemma 2.4. If $\left(A^{\mu}\right) \in \mathscr{P}$, then there exists a quantization $\left(\mathscr{K}_{0}, W_{0}, \Omega_{0}, U_{0}(\cdot)\right)$ over $(L, \sigma, V(\cdot))$ with $\Omega_{0} \in \mathscr{K}_{0}$ being the only $(\neq 0)$ vector in $\mathscr{K}_{0}$, which is invariant under $U_{0}(t)$ for all $t \in \mathbb{R}$. 
Proof. We extend the symplectic space $(L, \sigma)$ to a Hilbert space $\mathscr{H}_{P}$ such that the underlying real linear space is $L$, the imaginary part of the inner product is the symplectic form $\sigma$ and $V(\cdot)$ is a one-parameter strongly continuous group of unitary operators in $\mathscr{H}_{P}$ with positive generator. Define $\mathscr{H}_{I}^{+}=P_{+} \mathscr{H}_{I}$ and $\mathscr{H}_{I}^{-}=P_{-} \mathscr{H}_{I}$, then $\mathscr{H}_{P}=\mathscr{H}_{I}^{+} \oplus \overline{\mathscr{H}}_{I}^{-}$, where $\overline{\mathscr{H}}_{I}^{-}$is the Hilbert space conjugate to $\mathscr{H}_{I}^{-}$. The multiplication with complex numbers is defined by if $:=j f$, where $j$ is an operator in $\mathscr{H}_{I} ; j:=i\left(P_{+}-P_{-}\right)$. For all $f, g \in \mathscr{H}_{I}(f, g)_{P}=\operatorname{Re}(f, g)_{I}+i \sigma(f, g)$. Because $j V(t)=V(t) j$, the operators $V(t)$ are unitary in $\mathscr{H}_{P}$. From $i \partial_{t} V(t) f=B V(t) f$ for all $f \in D(B)$ in $\mathscr{H}_{E}$ follows $j \partial_{t} V(t) f=|B| V(t) f$ for all $f \in D(B)$; the operator $|B|$ is essentially self-adjoint in $\mathscr{H}_{P}$; so the $\mathscr{H}_{P}$-unitary group $V(\cdot)$ has $|\bar{B}|$ as its generator. $|\bar{B}|$ is positive, because $\sigma(|\bar{B}|) \subset \sigma(|B|) \subset(0, \infty)$ taking into account $\left(A^{\mu}\right) \in \mathscr{P}$. $\left(\sigma(|\bar{B}|)\right.$ is the spectrum of the operator $\overline{|B|}$ in $\mathscr{H}_{P}$ (or $\left.\mathscr{H}_{I}\right) ; \sigma(|B|)$ is the spectrum of the operator $|B|$ in $\mathscr{H}_{E}$.) So $0 \in \mathscr{H}_{P}$ is the only vector in $\mathscr{H}_{P}$, invariant under $V(t)$ for all $t \in \mathbb{R}$. The lemma is proven, if one considers the Fock quantization over $\left(\mathscr{H}_{P}, V(\cdot)\right)$.

Lemma 2.5. If $\left(A^{\mu}\right) \in \mathscr{P}$ and $(\mathscr{K}, W, \Omega, U(\cdot))$ is a quantization over $(L, \sigma, V(\cdot))$ with a unique vacuum and if the Weyl systems $(\mathscr{K}, W)$ and $\left(\mathscr{K}_{0}, W_{0}\right)$ (from Lemma 2.4) are unitarily equivalent, then the quantizations are unitarily equivalent.

Proof. The generating functional $E$ of the quantization $(\mathscr{K}, W, \Omega)$ has the form $E(f)=\exp \left(-\frac{1}{4}\|f\|_{P}^{2}+i F(f)\right)$ for all $f \in L=\mathscr{H}_{P}^{r}=\mathscr{H}_{I}^{r}[3] ; F$ is a $\mathbb{R}$-linear function on $L$ and invariant under $V(t)$ for all $t \in \mathbb{R}$. If $(\mathscr{K}, W)$ and $\left(\mathscr{K}_{0}, W_{0}\right)$ are unitarily equivalent, then $F$ is a continuous functional on $L$. From $0 \notin \sigma(\mid \overline{|B|})$ one deduces $F=0$ on $L$. Application of the G.N.S. theorem proves the lemma.

\section{The Interaction Hamiltonian in the Fock Space of the Free Field}

For the special case $A^{\mu}=0 \quad(\mu=0,1,2,3)$ one can construct $\mathscr{H}_{I}=W^{\frac{1}{2}}\left(\mathbb{R}^{3}\right) \oplus$ $W^{-\frac{1}{2}}\left(\mathbb{R}^{3}\right)$, the free one-particle space $\mathscr{H}_{P}$ and the Fock system $(\mathscr{K}, W, \Omega)$ over $\mathscr{H}_{P}$.

Let 2 be the set of real potentials $\left(A^{\mu}\right)$ on $\mathbb{R}^{3}$ with Fourier transforms $\tilde{A}^{\mu}$, such that the functions $\boldsymbol{p} \mapsto(1+|\boldsymbol{p}|) \tilde{A}^{\mu}(\boldsymbol{p})$ are integrable over $\mathbb{R}^{3}(\mu=0,1,2,3)$.

Lemma 3.1. If $\left(A^{\mu}\right) \in \mathscr{Q}$, then

1) $B_{\text {int }}$ with $D\left(B_{\text {int }}\right)=\mathscr{H}_{E}$ can be extended to a bounded operator $\bar{B}_{\text {int }}$ on $\mathscr{H}_{I}$.

2) The equation $i \partial_{t} f(t)=\left(\bar{B}_{0}+\bar{B}_{\text {int }}\right) f(t)$ has for all $f \in D\left(\bar{B}_{0}\right)$ a unique differentiable solution $f(\cdot)$ in $\mathscr{H}_{I}$ with $f(0)=f$. Define for all $f=f(0) \in D\left(\bar{B}_{0}\right), V(t) f=f(t)$; $V(\cdot)$ is a one-parameter strongly continuous group of bounded operators in $\mathscr{H}_{I}$.

3) $(V(t) f, V(t) g)_{Q}=(f, g)_{Q}$ for all $t \in \mathbb{R}$ and for all $f, g \in \mathscr{H}_{I}$.

Proof. 1) is proven by the same method as in the appendix of [1]. Define $\tilde{V}(t)=\sum_{n=0}^{\infty} R_{n}(t)$ with $R_{0}(t)=\mathbb{1}$ and $R_{n}(t)=\int_{0}^{t} B(s) R_{n-1}(s) d s(n \geqq 1)$ and $B(t)=-i e^{i \bar{B}_{0} t} \bar{B}_{\text {int }} e^{-i \bar{B}_{0} t}$.

The series is uniformly convergent in norm on any compact interval in $\mathbb{R}$; the function $t \mapsto \tilde{V}(t)$ is norm continuous. Because $\tilde{V}(t) D\left(\bar{B}_{0}\right) \subset D\left(\bar{B}_{0}\right), \quad V(t)=$ $\exp \left(-i \bar{B}_{0} t\right) \tilde{V}(t)$ represents the solution of equation 2$)$. The domain $D\left(B_{0}\right)$ is also 
left invariant by the operators $V(t)$; on $D\left(B_{0}\right)$ one shows 3) by direct computation; it then holds on $\mathscr{H}_{I}$ by continuity. We remark that the operators $V(t)$ are not linear in $\mathscr{H}_{P}$, because they do not commute with multiplication by $i$.

Lemma 3.2. If $\left(A^{\mu}\right) \in \mathscr{Q}$ and $V(\cdot)$ is the solution of the differential equation $\partial_{t} f(t)=$ $\left(\bar{B}_{0}+\bar{B}_{\text {int }}\right) f(t)$ in $\mathscr{H}_{1}$, then

1) for all $t \in \mathbb{R} P_{+} V(t) P_{+}$is a bounded, one-to-one operator from $\mathscr{H}_{I}^{+}$onto $\mathscr{H}_{I}^{+}$.

2) The mapping $t \mapsto\left(P_{+} V(t) P_{+}\right)^{-1} \phi$ is continuous for all $\phi \in \mathscr{H}_{I}^{+}$; the mapping $t \mapsto\left(P_{+} V(t) P_{+}\right)^{*}$ is strongly continuous.

3) For all $f \in \mathscr{H}_{I}^{+}\left(P_{+} V(t) P_{-}\right) *\left(P_{+} V(t) P_{+}\right) f-\left(P_{-} V(t) P_{-}\right) *\left(P_{-} V(t) P_{+}\right) f=0$.

Proof. The operator $P_{+} V(t) P_{+}$is invertible and Range $\left(P_{+} V(t) P_{+}\right)$is dense in $\mathscr{H}_{I}^{+}([4])$. For the inverse of the operator $P_{+} V(t) P_{+}$one finds $\left(P_{+} V(t) P_{+}\right)^{-1}=$ $\left(P_{+} V(t)^{-1} P_{+} V(t) P_{+}\right)^{-1} P_{+} V(t)^{-1} P_{+}$with $\left\|\left(P_{+} V(t)^{-1} P_{+} V(t) P_{+}\right)^{-1}\right\| \leqq 1$. Because $\left(P_{+} V(t) P_{+}\right)^{-1}$ is a bounded operator on Range $\left(P_{+} V(t) P_{+}\right)$, the range is the whole of $\mathscr{H}_{I}^{+}$. The continuity of the mapping $t \mapsto\left(P_{+} V(t) P_{+}\right)^{-1} \phi$ is now trivial. The mapping $t \mapsto \tilde{V}(t)^{*}$ is norm continuous, so $t \mapsto P_{+} e^{i \bar{B}_{0} t} \tilde{V}(t)^{*} P_{+}$is strongly continuous. The result 3) can be found in [4] or [5].

In the space $\mathscr{H}_{P}$ the one-parameter group $V(\cdot)$ of $\mathscr{H}_{P}^{r}$-bounded operators is symplectic (Lemma 3.1); so the operators $W_{t}(f)$, defined by $W_{t}(f)=W\left(V(t)^{-1} f\right)$ constitute for every time $t$ a Weyl system in the Fock space $\mathscr{K}_{\text {over }} \mathscr{H}_{P}$.

Lemma 3.3. Let $\left(A^{\mu}\right)$ be an element of 2 .

The one-parameter sympletic group $V(\cdot)$ in $\mathscr{H}_{P}$ is unitarily implementable in the Fock space $\mathscr{K}$ of the free field, if and only if the mapping $t \mapsto P_{-} V(t) P_{+}$is HilbertSchmidt continuous in $\mathscr{H}_{I}$.

Proof. Let $V(t)=U(V(t))\left(V(t)^{T} V(t)\right)^{1 / 2}$ be the polar decomposition of $V(t)$ in $\mathscr{H}_{P}^{r}$; $U(V(t))$ is an orthogonal operator and $V(t)^{T}$ is the adjoint of $V(t)$ in $\mathscr{H}_{P}^{r} . V(t)^{T}=$ $V(t)^{*}=-j V(t)^{-1} j ; V(t)^{*}$ is the adjoint of $V(t)$ in $\mathscr{H}_{I}$.

According to a result of Shale [6] it is necessary and sufficient for the oneparameter symplectic group in $\mathscr{H}_{P}$ to be unitarily implementable in the Fock space $\mathscr{K}$, that $t \mapsto U(V(t))$ is strongly continuous in $\mathscr{H}_{P}^{r}$ and $t \mapsto\left(V(t)^{T} V(t)\right)^{1 / 2}-\mathbb{1}$ is Hilbert-Schmidt (H.S.) continuous in $\mathscr{H}_{P}^{r}$. In our case the mapping $t \mapsto V(t)$ is strongiy continuous, so the strong continuity of $t \mapsto U(V(t))$ is automatically satisfied. A further simplification is obtained by observing that the H.S. continuity of the mapping $t \mapsto\left(V(t)^{T} V(t)\right)^{1 / 2}-\mathbb{1}$ in $\mathscr{H}_{P}^{r}=\mathscr{H}_{I}^{r}$ is equivalent to the H.S. continuity of the mapping $t \mapsto V(t)^{T} V(t)-\mathbb{1}$ in $\mathscr{H}_{I}$. The strong continuity of the function $t \mapsto V(t)$ entails the equivalence of the last statement with H.S. continuity of the mapping $t \mapsto P_{-} V(t) P_{+}-P_{+} V(t) P_{-}$on $\mathscr{H}_{I}$.

With Lemma 3.23$)$ one proves that the function $t \mapsto P_{+} V(t) P_{-}$is H.S. continuous in $\mathscr{H}_{I}$, if the function $t \mapsto P_{-} V(t) P_{+}$is H.S. continuous in $\mathscr{H}_{I}$.

The next lemma shows that it is sufficient to consider the Born term of the time-evolution $V(t)$.

Lemma 3.4. If $\left(A^{\mu}\right) \in \mathscr{Q}$, then the mapping $t \mapsto P_{-} V(t) P_{+}$is H.S. continuous in $\mathscr{H}_{I}$, if and only if the mapping $t \mapsto P_{-} R_{1}(t) P_{+}$is H.S. continuous in $\mathscr{H}_{I}$. 
Proof. Adapting the proof of Lemma 3 in [7] one can show that the H.S. continuity of the Born term $P_{-} R_{1}(t) P_{+}$is sufficient. To prove that this condition is also necessary, one observes that the function $t \mapsto P_{-} R_{1}(t) P_{+}$is the uniquely determined solution of the integral equation

$$
X(t)=C(t) A(t)^{-1}+\int_{0}^{t} X(s) D(s) A(t)^{-1} d s
$$

with $X(t) \in \mathscr{B}\left(\mathscr{H}_{I}^{+}, \mathscr{H}_{I}^{-}\right)$for all $t \in \mathbb{R}, t \mapsto X(t)$ strongly continuous and $X(0)=0$. The coefficients of the equation are defined by $A(t)=P_{+} \tilde{V}(t) P_{+}, C(t)=P_{-} \tilde{V}(t) P_{+}-$ $\int_{0}^{t} P_{-} B(s) P_{-} \tilde{V}(s) P_{+} d s$ and $D(t)=P_{+} B(t) \tilde{V}(t) P_{+} \cdot$

The function $t \mapsto\left(A(t)^{-1}\right)^{*}$ is strongly continuous and from our assumption the function $t \mapsto C(t)$ is H.S. continuous; so the function $t \mapsto \varrho_{0}(t)=C(t) A(t)^{-1}$ is H.S. continuous. Also the function $t \mapsto D(t)^{*}$ is strongly continuous; so the functions $t \mapsto \varrho_{n}(t)=\int_{0}^{t} \varrho_{n-1}(s) D(s) A(t)^{-1} d s$ are H.S. continuous. On the interval $[-a, a]$ $(a>0)$ the series $\sum_{n=0}^{\infty} \varrho_{n}(t)$ is uniformly convergent in $\mathscr{I}_{2}\left(\mathscr{H}_{I}^{+}, \mathscr{H}_{I}^{-}\right)$(the Banach space of H.S. operators from $\mathscr{H}_{I}^{+}$to $\mathscr{H}_{I}^{-}$with H.S. norm). This proves that the function $t \mapsto \sum_{n=0}^{\infty} \varrho_{n}(t)$ is H.S. continuous on $[-a, a]$. The series satisfies the integral equation; by uniqueness of the solution $P_{-} R_{1}(t) P_{+}=\sum_{n=0}^{\infty} \varrho_{n}(t)$ for all $t \in \mathbb{R}$.

Theorem 3.5. Let $\left(A^{\mu}\right)$ be an element of $\mathscr{Q}$.

The one-parameter symplectic group $V(\cdot)$ in $\left(\mathscr{H}_{P}^{r}, \sigma\right)$ is unitarily implementable in the Fock space $\mathscr{K}$ of the free field, if and only if $\boldsymbol{A}=0$ on $\mathbb{R}^{3}$ and for all $t \in \mathbb{R}$ $Q_{0}(t)$ is finite and the function $t \mapsto Q_{0}(t)$ is continuous at $t=0$.

Proof. By applying Fubini's theorem twice one obtains for all $t \in \mathbb{R}$

with

$$
\left\|P_{-} R_{1}(t) P_{+}\right\|_{2}=\int\left|K_{t}\left(\boldsymbol{p}, \boldsymbol{p}^{\prime}\right)\right|^{2} \frac{d \boldsymbol{p} d \boldsymbol{p}^{\prime}}{2 \omega(\boldsymbol{p}) 2 \omega\left(\boldsymbol{p}^{\prime}\right)}
$$

$$
\begin{aligned}
K_{t}\left(\boldsymbol{p}, \boldsymbol{p}^{\prime}\right)= & i\left(-1+\exp \left(-i\left(\omega(\boldsymbol{p})+\omega\left(\boldsymbol{p}^{\prime}\right)\right) t\right)\right) 2 \omega\left(\boldsymbol{p}^{\prime}\right) B_{\mathrm{int}}^{-}\left(\boldsymbol{p}, \boldsymbol{p}^{\prime}\right) /\left(\omega(\boldsymbol{p})+\omega\left(\boldsymbol{p}^{\prime}\right)\right), \\
B_{\mathrm{int}}^{-+}\left(\boldsymbol{p}, \boldsymbol{p}^{\prime}\right)= & \left(-\omega\left(\boldsymbol{p}^{\prime}\right)^{-1} \omega(\boldsymbol{p})+1\right) \tilde{A_{0}}\left(\boldsymbol{p}-\boldsymbol{p}^{\prime}\right)+\omega(\boldsymbol{p})^{-1} \tilde{\boldsymbol{A}} * \tilde{\boldsymbol{A}}\left(\boldsymbol{p}-\boldsymbol{p}^{\prime}\right) \\
& -\omega\left(\boldsymbol{p}^{\prime}\right)^{-1}\left(\boldsymbol{p}+\boldsymbol{p}^{\prime}\right) \cdot \tilde{\boldsymbol{A}}\left(\boldsymbol{p}-\boldsymbol{p}^{\prime}\right) .
\end{aligned}
$$

If there exists a point $p \in \mathbb{R}^{3}$ with $\tilde{A}(p) \neq 0$, then one obtains a contradiction with $K_{t} \in L^{2}\left(\mathbb{R}^{3} \times \mathbb{R}^{3}, \frac{d \boldsymbol{p}}{\omega(\mathbf{p})} \frac{d \boldsymbol{p}^{\prime}}{\omega\left(\mathbf{p}^{\prime}\right)}\right)$ Setting $\boldsymbol{A}=0$ on $\mathbb{R}^{3}$ the H.S. continuity of the function $t \mapsto P_{-} R_{1}(t) P_{+}$implies the continuity of the mapping $t \mapsto Q_{0}(t)$ at $t=0$. Remark. For spin one-half particles this theorem was generalized independently in [8] and [9]. 
Acknowledgements. This work was part of a doctoral thesis at the II. Institut für Theoretische Physik der Universität Hamburg. I am indebted to Prof. G. Roepstorff for his supervision and his stimulating interest in this work. I am grateful for the hospitality at Lehrstuhl E der R.W.T.H. Aachen.

The Deutscher Akademischer Austauschdienst, the Freie und Hansestadt Hamburg and the Bundesministerium für Forschung und Technologie financially supported this investigation.

\section{References}

1. Schroer, B., Seiler, R., Swieca, J. A. : Phys. Rev. D2, 2927-2937 (1970)

2. Thoe, D.: Arch. Rat. Mech. Anal. 22, $364-406$ (1966)

3. Weinless, M.: J. Funct. Anal. 4, 350-379 (1969)

4. Lundberg, L.-E.: Commun. math. Phys. 31, 295-316 (1973)

5. Seiler, R.: Commun. math. Phys. 25, 127-150 (1972)

6. Shale, D. : Trans. Amer. Math. Soc. 103, 149-167 (1962)

7. Bongaarts, P. J.M.: Ann. Phys. 56, 108-139 (1970)

8. Fredenhagen, K.: Thesis, Hamburg 1976

9. Ruijsenaars, S. N.M.: Thesis, Leiden 1976

Communicated by R. Haag

Received June 10, 1976 
